\title{
Quadratic Forms in Normal RVs: Theory and Applications to OSTBC over Hoyt Fading Channels
}

\author{
George A. Ropokis, Student Member, IEEE, Athanasios A. Rontogiannis, Member, IEEE, \\ and P. Takis Mathiopoulos, Senior Member, IEEE
}

\begin{abstract}
In this paper, the statistics of quadratic forms in normal random variables (RVs) are studied and their impact on performance analysis of wireless communication systems is explored. First, a chi-squared series expansion is adopted to represent the probability density function of a quadratic form in normal RVs and novel series truncation error bounds are derived, which are much tighter compared to already known ones. Secondly, it is theoretically shown that when an orthogonal space time block coding (OSTBC) transmission scheme is used, the signal to noise ratio (SNR) at the receiver under various fading conditions can be expressed as a quadratic form in normal RVs. Capitalizing on these results, a thorough error probability and capacity analysis is presented for the performance of OSTBC systems over Nakagami- $q$ (Hoyt) fading channels. For all error probability and capacity performance criteria considered, simple, closed-form truncation error bounds expressions are derived, which avoid the use of infinite sums and complicated functions. The proposed theoretical analysis is validated through extensive Monte Carlo simulations.
\end{abstract}

Index Terms-Quadratic forms in normal RVs, OSTBC, MIMO, Nakagami- $q$ (Hoyt) distribution, fading channels, performance analysis.

\section{INTRODUCTION}

$\mathbf{P}$ ERFORMANCE analysis of wireless mobile digital communication systems depends mainly on two factors, namely the channel fading statistics and the utilized diversity scheme. In such communications systems, the transmitted signal is impaired by fading resulting in severe performance degradation. To combat fading, diversity schemes can be applied at the transmitter or the receiver by properly combining multiple replicas of the transmitted signal. In several applications, fading and diversity are such that the statistics of a quadratic form in normal random variables (RVs) need to be evaluated. This necessity has motivated our current research

Manuscript received July 26, 2007; revised November 15, 2007 and February 11, 2007; accepted February 18, 2008. The associate editor coordinating the review of this manuscript and approving it for publication was S. Rajan.

This paper is part of the 03ED838 research project, implemented within the framework of the "Reinforcement Programme of Human Research Manpower" (PENED) and co-financed by National and Community Funds $(75 \%$ from E.U.-European Social Fund and 25\% from the Greek Ministry of Development-General Secretariat of Research and Technology). Part of this work has been presented in GLOBECOM 2007.

G. A. Ropokis is with the Dept. of Computer Engineering \& Informatics, University of Patras, 26500, Rio, Greece and the Institute for Space Applications and Remote Sensing, National Observatory of Athens, 15236, Greece (e-mail: ropokis@space.noa.gr)

A. A. Rontogiannis and P. T. Mathiopoulos are with the Institute for Space Applications and Remote Sensing, National Observatory of Athens, 15236, Greece (e-mail: \{tronto, mathio\}@space.noa.gr).

Digital Object Identifier 10.1109/T-WC.2008.070830 effort since, in general, analytical closed-form expressions for the probability density function (PDF) and the cumulative distribution function (CDF) of a quadratic form in normal RVs are not available. These functions are usually represented by certain infinite series expansions [1], whose convergence is controled by properly selecting the values of their parameters. When a series representation is used, bounds on the truncation error, i.e., on the error resulting after retaining a number of leading series terms, are becoming of major importance. Closed-form expressions of truncation error bounds for various series expansions related to quadratic forms in normal RVs have been reported several years ago in the statistical literature [2], [3].

An important application, where a quadratic form in normal $\mathrm{RVs}$ appears, is in studying the performance of orthogonal space-time block coding (OSTBC) over various fading channels. In recent years OSTBC is becoming increasingly popular as an efficient transmit diversity technique to combat fading in wireless communications [4]. This approach offers full spatial diversity and maximum likelihood performance with linear decoding complexity. In the past, several performance analysis results have been reported for OSTBC operating over various classical fading channels, including Rayleigh [5], Nakagami-m [6], [7], and Nakagami- $n$ (Rice) [8]. Another distribution, which has recently received increased attention in modeling fading channels, is the Nakagami- $q$ (Hoyt) distribution [9]. Studies have shown that the Hoyt fading channel model provides a very accurate fit to experimental channel measurements in various telecommunications applications. For instance, in [10] this model has been used in outage analysis of cellular mobile radio systems, in [11] the capacity of Hoyt fading channels has been studied and more recently, in [12] an error performance analysis of $M$-ary modulation schemes in Hoyt fading channels was presented. Similarly, the Hoyt distribution can be considered as an accurate fading model for satellite links with strong ionospheric scintillation [13]. However, regarding the performance of OSTBC schemes over Hoyt fading channels, very few studies have been published in the open technical literature. Such studies usually have dealt with specific performance criteria, e.g., ergodic capacity [14], and information outage probability [15].

A well known approach that could be used to analyze the performance of OSTBC over Hoyt fading channels is the moment generating function (MGF) method [9]. It is not difficult to show that for only special cases of specific fading parameter values, the MGF method leads to closed 
form expressions for various performance metrics. However, for the general problem we are dealing with in this paper, the MGF method fails to provide a compact and mathematically tractable framework, which enables accurate performance analysis.

Motivated by the above, in this paper, a new approach for the performance evaluation of OSTBC wireless communication systems is introduced and analyzed. This approach is based on the analysis of the statistics of quadratic forms in normal RVs. To this end, we first consider the formulation of central quadratic forms in normal RVs, i.e., quadratic forms where the involved random variables are zero mean, which may be statistically independent or not. Following that, the chi-squared series expansion is used to represent the PDF and $\mathrm{CDF}$ of central positive definite quadratic forms [1]. The main advantages of our approach are its simplicity, mathematical tractability and fast convergence. Based on these representations, new analytical closed-form expressions of truncation error bounds are derived for both the PDF and the CDF, which are much tighter as compared to already known ones [2], [3]. The bounds are derived following a novel approach, while their analytical expressions are quite simple allowing for further mathematical manipulations.

In the sequel, a detailed performance analysis of OSTBC operating over not necessarily identical Hoyt distributed fading channels is presented. First, it is shown that the problem of evaluating the performance of OSTBC reduces to the analysis of the distribution of a central, positive definite quadratic form in normal RVs. Hence the previously mentioned framework analysis can be directly applied in solving this problem. Following that an error probability as well as a capacity analysis are conducted based on the chi-squared series representation. Closed-form infinite series expressions for the bit error probability (BEP) and the symbol error probability (SEP) of various modulation schemes are presented and novel tight truncation error bounds for these expressions are given in a mathematically simple form. A similar analysis is also presented for the capacity, including the information outage probability and the ergodic capacity, of OSTBC over Hoyt fading channels. The theoretical analysis has been also verified by means of extensive computer simulated performance evaluation results.

The paper is organized as follows. After this introduction, in Section II, quadratic forms in normal RVs are defined for both independent and correlated RVs. In Section III, the chi-squared series expansion is utilized and novel truncation error bounds for the PDF and CDF of a quadratic form in normal RVs are derived. Section IV, studies OSTBC for fading channels, while a detailed performance analysis of OSTBC over Hoyt fading channels is presented in Section V. Various performance results (theoretical and computer simulated) are presented and discussed in Section VI. The conclusion of the paper can be found in Section VII.

\section{QUADRATIC FORMS IN NORMAL RVS}

Let $\mathbf{A}$ be an $n \times n$ real symmetric matrix and $\mathbf{x}$ an $n \times$ 1 vector of zero mean normal RVs with a positive definite covariance matrix $\mathbf{C}=\operatorname{Cov}(\mathbf{x})$. Then a quadratic form in central normal RVs is defined as follows $[1]^{1}$

$$
Q \triangleq \mathbf{x}^{T} \mathbf{A} \mathbf{x}
$$

where $(\cdot)^{T}$ denotes transposition. It should be emphasized that irrespective of the covariance matrix $\mathbf{C}, Q$ can be equivalently written as a function of zero mean uncorrelated normal RVs. Indeed, by making the transformation

$$
\mathbf{z}=\mathbf{C}^{-1 / 2} \mathbf{x}
$$

where $\mathbf{C}^{1 / 2}$ denotes a square root of $\mathbf{C}$, (1) becomes

$$
Q=\mathbf{z}^{T} \mathbf{R z}
$$

where $\mathbf{R}=\mathbf{C}^{T / 2} \mathbf{A} \mathbf{C}^{1 / 2}$. Substituting the real symmetric matrix $\mathbf{R}$ in (3) by its eigenvalue decomposition, i.e., $\mathbf{R}=$ $\mathbf{U}^{T} \boldsymbol{\Lambda} \mathbf{U}$, where $\mathbf{U}$ is an orthonormal matrix and $\boldsymbol{\Lambda}$ a diagonal matrix containing the real eigenvalues of $\mathbf{R}$, yields

$$
Q=\mathbf{y}^{T} \boldsymbol{\Lambda} \mathbf{y}
$$

where $\mathbf{y}=\mathbf{U z}$. Obviously, $\mathbf{y}$ has zero mean i.e., $E[\mathbf{y}]=$ 0 , and covariance $\operatorname{Cov}(\mathbf{y})=\mathbf{I}$, where $\mathbf{I}$ denotes the identity matrix. Equivalently, the above equation can be expressed as

$$
Q=\sum_{i=1}^{n} \lambda_{i} y_{i}^{2}
$$

where for $i=1,2, \ldots, n, \lambda_{i}$ are the real eigenvalues of $\mathbf{R}$ and $y_{i}$ zero mean independent normal RVs. When matrix $\mathbf{A}$ is positive definite, then $\lambda_{i}>0, \forall i$, and $Q$ is characterized as a central, positive definite quadratic form, the analysis of which will be the main focus of this work.

As it will be shown in Section IV quadratic forms in normal RVs show up in several applications related to performance evaluation of wireless communication systems. Thus, in order to obtain expressions for the various performance criteria, the PDF and CDF of such forms must be determined in a mathematically tractable form, which is amenable to further manipulations. In the following section the PDF and CDF of $Q$ in (5) are expressed based on the chi-squared series expansion and tight upper bounds on the series' truncation errors are derived.

\section{QUADRATIC FORMS STATISTICS}

In general, there is no known analytical expression for the $\mathrm{PDF}$ and CDF of $Q$ in (5). However, various infinite series expansions have been proposed to evaluate the statistics of quadratic forms in normal RVs [1]. One such a representation is the chi-squared series expansion which offers mathematical tractability and fast convergence. Using a chi-squared series expansion the PDF of $Q$ is expressed as follows [1, pp. 115 123]

$$
p_{Q}(x)=\sum_{k=0}^{\infty} c_{k} f\left(x, 2 \beta, \frac{n}{2}+k\right)
$$

where

$$
f(x, b, l)=\frac{x^{l-1} \exp \left(-\frac{x}{b}\right)}{b^{l} \Gamma(l)}
$$

\footnotetext{
${ }^{1}$ If $\mathbf{A}$ is not symmetric, symmetry can be imposed by writing $Q$ in the equivalent form $Q=\mathbf{x}^{T} \frac{\left(\mathbf{A}+\mathbf{A}^{T}\right)}{2} \mathbf{x}$.
} 
while its $\mathrm{CDF}$ is given by

$$
P_{Q}(x)=\sum_{k=0}^{\infty} c_{k} F\left(x, 2 \beta, \frac{n}{2}+k\right)
$$

with

$$
F(x, b, l)=\frac{1}{\Gamma(l)} \gamma\left(l, \frac{x}{b}\right)
$$

In the previous expressions, $\Gamma(\cdot)$ is the gamma function $[16$, eq. (6.1.1)],$\gamma(\cdot, \cdot)$ the lower incomplete gamma function [16, eq. (6.5.2)] and $\beta$ is an arbitrary positive parameter controlling the convergence of the series. More specifically as stated in [3], for any choice of $\beta>0$, the uniform convergence of (6) and (8) in any finite interval on the positive semiaxis can be ensured. Additionally, if $\beta$ is chosen such that $\beta<2 \min \left\{\lambda_{i}\right\}$, the PDF and CDF series converge uniformly for all ${ }^{i} x>0$. According to the analysis in [2], by choosing $\beta$ as

$$
\beta=\frac{2 \max _{i}\left\{\lambda_{i}\right\} \min _{i}\left\{\lambda_{i}\right\}}{\max _{i}\left\{\lambda_{i}\right\}+\min _{i}\left\{\lambda_{i}\right\}}
$$

not only uniform convergence of (6) and (8) for all $x>0$ is guaranteed, but also a sufficiently high rate of convergence of the series (6) and (8) is achieved. Hence in [2] selection of $\beta$ as in (10) is proposed, which is also adopted in our simulations. It must be noted that, in general, for any choice of $\beta$ the rate of convergence depends on the spread of the values $\lambda_{i}$, i.e., on the ratio $\left[\max _{i}\left\{\lambda_{i}\right\} / \min _{i}\left\{\lambda_{i}\right\}\right]$ with small spreads resulting in faster convergence. The coefficients $c_{k}$ of the expansions (6) and (8) can be obtained recursively as

$$
c_{k}=\frac{1}{2 k} \sum_{l=0}^{k-1} d_{k-l} c_{l} \text { for } k>0
$$

with

$$
c_{0}=\prod_{i=1}^{n}\left(\frac{\beta}{\lambda_{i}}\right)^{1 / 2} \text { and } d_{k}=\sum_{i=1}^{n}\left(1-\frac{\beta}{\lambda_{i}}\right)^{k} .
$$

Although (11) and (12) provide an efficient way to compute the coefficients of the chi-squared series, here, an alternative expression proposed in [3] will be adopted

$$
c_{k}=c_{0} E\left[Z^{k}\right] /\left(2^{k} k !\right)
$$

where $E\left[Z^{k}\right]$ is the $k$-th moment of a quadratic form $Z$ defined as

$$
Z \triangleq \sum_{i=1}^{n} \eta_{i} y_{i}^{2}, \quad \text { with } \quad \eta_{i}=1-\frac{\beta}{\lambda_{i}} .
$$

As it will become clear in the following subsection, these expressions are more convenient in deriving truncation error bounds of chi-squared series.

\section{A. Truncation error bounds}

In practice, a truncated version of the infinite series (6) and (8) is used. Thus, in order to assess the accuracy of the truncated series, derivation of bounds on the truncation error becomes extremely important. Such bounds provide an indication of the number of series terms that must be kept in order to achieve a desired accuracy. Let's first consider the PDF from (6), and assume that $N+1$ series terms are retained.
To obtain a truncation error bound the following quantity must be properly bounded [3]

$$
e(x)=\sum_{k=N+1}^{\infty}\left|c_{k}\right| f\left(x, 2 \beta, \frac{n}{2}+k\right)
$$

where $|\cdot|$ denotes the absolute value. According to (13), to obtain a bound on $\left|c_{k}\right|$, the amplitude of the moments of $Z$ must be properly bounded. In [3], by considering a new quadratic form as

$$
W=\sum_{i=1}^{n}\left|\eta_{i}\right| y_{i}^{2}
$$

and using the inequality

$$
\left|E\left[Z^{k}\right]\right| \leq E\left[W^{k}\right] \leq \max _{i}\left|\eta_{i}\right|^{k} E\left[\left(\sum_{i=1}^{n} y_{i}^{2}\right)^{k}\right]
$$

the following bound on $\left|c_{k}\right|$ has been proposed

$$
\left|c_{k}\right| \leq c_{0}\left(\max _{i}\left\{\left|\eta_{i}\right|\right\}\right)^{k} \Gamma\left(\frac{n}{2}+k\right) /\left[k ! \Gamma\left(\frac{n}{2}\right)\right] .
$$

However, extensive simulation results have shown that the bound of (18) can be extremely loose (see, e.g., Fig. 1). In the applications of interest, as described in Section IV, the number of terms in the quadratic form is even. By taking advantage of this special property, a much tighter upper bound can be derived. Indeed, let us first rewrite $W$ as

$$
W=\sum_{i=1}^{n} \xi_{i} u_{i}^{2}
$$

where $\xi_{i}$ and $u_{i}$ are proper permutations of $\left|\eta_{i}\right|$ and $y_{i}$ respectively, such that $\xi_{i}$ are in decreasing order, for $i=1,2, \ldots, n$. The basic idea here is to formulate a new quadratic form, $V$, which results from $W$ by grouping in pairs successive squared normal RVs $u_{i}^{2}$, i.e.,

$$
V=\sum_{i=1}^{n / 2} \xi_{2 i-1}\left(u_{2 i-1}^{2}+u_{2 i}^{2}\right) .
$$

Comparing (19) and (20), clearly $W \leq V$ and thus $E\left[W^{k}\right] \leq$ $E\left[V^{k}\right] \forall k$. Furthermore and most importantly the moments of $V$ can be now easily obtained using its moment generating function (MGF), given by

$$
M_{V}(t)=\prod_{i=1}^{n / 2} \frac{1}{1-2 \xi_{2 i-1} t} .
$$

By employing partial fractions and assuming that all $\xi_{2 i-1}$ are distinct, (21) is expressed as:

$$
M_{V}(t)=\sum_{i=1}^{n / 2} A_{i} \frac{1}{1-2 \xi_{2 i-1} t}
$$

where

$$
A_{i}=\xi_{2 i-1}^{n / 2-1} \prod_{\substack{l=1 \\ l \neq i}}^{n / 2} \frac{1}{\xi_{2 i-1}-\xi_{2 l-1}}
$$


Noting that (22) corresponds to the MGF of a RV that is a mixture of gamma distributed RVs, it can be easily shown that the moments of $V$ can be expressed as:

$$
E\left[V^{k}\right]=\sum_{i=1}^{n / 2} A_{i} k !\left(2 \xi_{2 i-1}\right)^{k} .
$$

Since $\left|E\left[Z^{k}\right]\right| \leq E\left[W^{k}\right] \leq E\left[V^{k}\right]$, from (13), (23), and (24) the following very simple bound for $\left|c_{k}\right|$ can be obtained

$$
\left|c_{k}\right| \leq c_{0} \sum_{i=1}^{n / 2} \Delta_{i} \xi_{2 i-1}^{n / 2+k-1}, \text { with } \Delta_{i}=\prod_{\substack{l=1 \\ l \neq i}}^{n / 2} \frac{1}{\xi_{2 i-1}-\xi_{2 l-1}} .
$$

A tight truncation error bound, $e_{b}(x)$, of the PDF series (6) can now be defined by substituting (25) in the right hand side (RHS) of (15), i.e.,

$$
e_{b}(x)=\sum_{k=N+1}^{\infty} \sum_{i=1}^{n / 2} \Delta_{i} c_{0} \xi_{2 i-1}^{n / 2+k-1} f\left(x, 2 \beta, \frac{n}{2}+k\right) .
$$

Defining the series

$$
d(x, a, b) \triangleq \sum_{p=0}^{\infty} a^{p} f(x, b, p+1)
$$

from (7) it can be easily shown that

$$
d(x, a, b)=\frac{\exp \left[\frac{-(1-a) x}{b}\right]}{b} .
$$

Furthermore, it can be proven that the series in (27) converges uniformly for $a>0$, as long as $a<1$. Hence by selecting $\beta$ as $\beta<2 \min _{i}\left\{\lambda_{i}\right\}$, the condition $\xi_{2 i-1}<1$ for all $i$ is satisfied and the uniform convergence of (6) and (27) can be ensured. The truncation error bound is then expressed in closed-form as

$$
e_{b}(x)=c_{0} \sum_{i=1}^{n / 2} \Delta_{i} g\left(x, \xi_{2 i-1}, 2 \beta, N+\frac{n}{2}-1\right)
$$

where

$$
g(x, a, b, m)=d(x, a, b)-\sum_{k=0}^{m} a^{k} f(x, b, k+1) .
$$

Additionally, a truncation error bound of the CDF series (8) can now be derived from (30) by simple integration, yielding

$$
\varepsilon_{b}(x)=c_{0} \sum_{i=1}^{n / 2} \Delta_{i} G\left(x, \xi_{2 i-1}, 2 \beta, N+\frac{n}{2}-1\right)
$$

where

$$
G(x, a, b, m)=\int_{0}^{x}\left[d(t, a, b)-\sum_{k=0}^{m} a^{k} f(t, b, k+1)\right] d t .
$$

Using the incomplete gamma function $\gamma(\cdot, \cdot)$ the last equation is rewritten as

$$
G(x, a, b, m)=\frac{1-\exp \left[-\frac{(1-a) x}{b}\right]}{1-a}-\sum_{k=0}^{m} \frac{a^{k} \gamma\left(k+1, \frac{x}{b}\right)}{\Gamma(k+1)} .
$$

Although (29) and (31) were obtained using the reasonable assumption that all $\xi_{2 i-1}$ are distinct, similar expressions can be derived for non-distinct $\xi_{2 i-1}$ as shown in the Appendix. Notice also that (29) and (31) comprise simple elementary functions only. The importance of this property will become evident in the following sections, where the application of central, positive definite quadratic forms in normal RVs in the performance analysis of wireless communications systems is studied and (29), (31) will be used to assess the estimation accuracy of several infinite series expansions.

\section{OSTBC IN FADING CHANNELS}

Let us consider an OSTBC multiple-input multiple-output (MIMO) system with $n_{t}$ transmit and $n_{r}$ receive antennae. The symbols to be transmitted are organized in blocks and are properly coded in space and time so that the original MIMO system is transformed in a number of parallel single-input single-output (SISO) systems achieving maximal diversity. It can then be shown that the signal to noise ratio (SNR) per bit at the output of the system is expressed as [17]

$$
\gamma_{O S T B C}=\frac{\|\mathbf{H}\|_{F}^{2}}{R n_{t}} \gamma_{t}
$$

where $R$ is the rate of the space-time code and $\gamma_{t}$ is the SNR at the transmitter side, defined as the ratio of the transmitted energy per bit over the noise density. In addition,

$$
\|\mathbf{H}\|_{F}^{2}=\sum_{p=1}^{n_{r}} \sum_{j=1}^{n_{t}}\left|h_{p, j}\right|^{2}
$$

is the square of the Frobenius norm of the $n_{r} \times n_{t}$ channel matrix $\mathbf{H}$, and $\left|h_{p, j}\right|$ stands for the amplitude of the complex fading coefficient between the $j$ th transmit and $p$ th receive antenna. Under various fading models (e.g., Rayleigh, Ricean, or Nakagami- $q$ ), the real and imaginary parts of $h_{p, j}$ correspond to normal RVs. Consequently, from (34) and (35), the SNR is expressed as a quadratic form in normal RVs with an even number of terms equal to $2 n_{t} n_{r}$.

In this paper, the Nakagami- $q$ (Hoyt) fading scenario, for which only a few performance analysis results exist in the open technical literature is considered. This is probably due to the fact that in most previous efforts the Hoyt PDF was employed directly, which is rather difficult to manipulate. Instead, in this analysis we exploit the fact that when $\left|h_{p, j}\right|$ is Hoyt distributed, the real and imaginary parts of $h_{p, j}$ are zero-mean normal RVs with, in general, different variances ${ }^{2}$.

Thus, the SNR given in (34) is a central, positive definite quadratic form in normal RVs, and all the results presented

${ }^{2}$ Let $\theta$ and $\phi$ be zero-mean normal RVs with variances $\sigma_{\theta}^{2}$ and $\sigma_{\phi}^{2}$ respectively and $h=\theta+j \phi$. Then, $|h|$ is Hoyt distributed and its PDF is given by [9]

$$
p_{|h|}(x)=\frac{\left(1+q^{2}\right) x}{q \Omega} \exp \left[-\frac{\left(1+q^{2}\right)^{2} x^{2}}{4 q^{2} \Omega}\right] I_{0}\left(\frac{\left(1-q^{4}\right) x^{2}}{4 q^{2} \Omega}\right)
$$

where $I_{0}(\cdot)$ is the zeroth order modified Bessel function of the first kind [16, eq. (9.6.16)] and the parameters $q$ and $\Omega$ are related to $\sigma_{\theta}^{2}$ and $\sigma_{\phi}^{2}$ as

$$
\sigma_{\theta}^{2}=\frac{\Omega}{1+q^{2}} \quad \text { and } \quad \sigma_{\phi}^{2}=\frac{\Omega q^{2}}{1+q^{2}} .
$$


in the previous section apply directly to $\gamma_{O S T B C}$. More specifically, the quadratic form in (34) consists of $2 n_{t} n_{r}$ squared zero-mean normal RVs with, in general, different variances $\sigma_{i}^{2}, i=1,2, \ldots, 2 n_{t} n_{r}$. Then, from (5) and (34), $\lambda_{i}$ can be defined as $\lambda_{i}=\sigma_{i}^{2} \gamma_{t} / R n_{t}, i=1,2, \ldots, 2 n_{t} n_{r}$. The PDF and CDF of $\gamma_{O S T B C}$ can be obtained by using (6) and (8) respectively, where now the coefficients $c_{k}$ are suitably defined according to (11) and (12) and $\lambda_{i}$ given as above. Consequently, several performance analysis criteria of OSTBC over Hoyt fading can be now derived. Moreover, the accuracy of the proposed metrics can be evaluated through appropriate closed-form truncation error bounds.

In the following section, an error probability analysis for various modulation schemes, as well as a capacity analysis for OSTBC schemes over Hoyt fading is presented. Note that the Hoyt RVs corresponding to the fading coefficients may be correlated ${ }^{3}$. In such case, a suitable transformation of the resulting quadratic form in (34) can be applied to obtain a quadratic form with independent normal RVs, as explained in Section II. Hence, the performance analysis presented in the next section applies to both independent as well as correlated, not necessarily identical Hoyt fading channel coefficients in OSTBC MIMO systems.

\section{Performance Analysis of OStBC over Hoyt FADING CHANNELS}

In this section a detailed error probability and capacity analysis are presented for OSTBC schemes over Hoyt fading channels, following the approach developed in the previous sections. Based on the chi-squared series representation, novel expressions for the symbol and bit error probabilities of various modulation schemes as well as the information outage probability and the ergodic capacity are presented. For all these performance criteria, simple expressions of the truncation error bounds, which avoid the use of infinite sums and complicated functions, are also derived.

\section{A. Error Probability Analysis}

For the error probability analysis, the calculation of integrals of the form

$$
\mathcal{P}=\int_{0}^{\infty} p_{Q}(\gamma) P(\gamma) d \gamma
$$

is required, where $P(\cdot)$ is a function related to the modulation scheme. A common approach for the calculation of such types of integrals is by using the MGF of $Q$ [9]. It can be shown that by following this approach integrals of the form

$$
\mathcal{M}=\int_{0}^{\theta} \prod_{i=1}^{2 n_{t} n_{r}} \frac{1}{\left(1+2 \lambda_{i} \frac{g}{\sin ^{2} \phi}\right)^{1 / 2}} d \phi
$$

need to be evaluated, where $\theta$ and $g$ are modulation specific constants. In special cases, depending on the values of the model parameters $\lambda_{i}$, the integral $\mathcal{M}$ can be evaluated in closed form. Specifically, for this to happen, all distinct parameters $\lambda_{i}$ should appear with even multiplicities, leading

\footnotetext{
${ }^{3}$ Here, correlation among Hoyt RVs is meant as correlation among their constituent normal RVs.
}

to integer powers in the denominator of the integrand in (37), i.e.,

$$
\mathcal{M}=\int_{0}^{\theta} \prod_{\lambda_{i} \in \mathcal{S}} \frac{1}{\left(1+2 \lambda_{i} \frac{g}{\sin ^{2} \phi}\right)^{m_{i}}} d \phi
$$

where $\mathcal{S}$ is the set of distinct values of the parameters $\lambda_{i}$ and $m_{i}$ are integers. It must be noted that when $m_{i}$ are integers, the PDF of $Q$ has a closed form expression, which can also be obtained by properly manipulating (6), (7), (13) and (14). For these values of $m_{i}$, the PDF and the MGF approaches, given by (36) and (37) respectively, are equivalent. Nevertheless, to the best of our knowledge, no closed form solution exists for (37) when the model parameters $\lambda_{i}$ take arbitrary values, as is the case in the problem under consideration in this work. To derive closed form expressions for various performance metrics, we propose the substitution of $p_{Q}(x)$ in (36) by the infinite series representation given in (6). Then, (36) is rewritten as

$$
\mathcal{P}=\sum_{k=0}^{\infty} c_{k} \int_{0}^{\infty} f\left(\gamma, 2 \beta, \frac{n}{2}+k\right) P(\gamma) d \gamma
$$

where $f(\cdot, \cdot, \cdot)$ is given by (7). Additionally, using (29) bounds on the truncation error of the SEP and BEP series can be obtained by evaluating the integral

$$
\mathcal{E}=\int_{0}^{\infty} e_{b}(\gamma) P(\gamma) d \gamma .
$$

1) SEP Expressions: In the following SEP expressions and the corresponding truncation error bounds are derived for various modulation schemes.

$M$-PAM: For an $M$-PAM modulation scheme, $P(\gamma)$ is given by [9]

$$
P(\gamma)=\delta \mathcal{Q}(a \sqrt{\gamma})
$$

where $\mathcal{Q}(\cdot)$ is the Gaussian $Q$-function, [9, eq. (4.1)] and $a$, $\delta$ are given as

$$
a=\sqrt{\frac{6 \log _{2} M}{M^{2}-1}}, \quad \delta=2 \frac{M-1}{M} .
$$

For integer $m$, it is shown in [9, p. 127$]$ that

$$
\begin{aligned}
L(a, b, m) & =\int_{0}^{\infty} f(\gamma, b, m) \mathcal{Q}(a \sqrt{\gamma}) d \gamma \\
& =\frac{1}{2}\left[1-\mu \sum_{l=0}^{m-1}\left(\begin{array}{c}
2 l \\
l
\end{array}\right)\left(\frac{1-\mu^{2}}{4}\right)^{l}\right]
\end{aligned}
$$

where

$$
\mu=\sqrt{\frac{a^{2} b}{2+a^{2} b}} .
$$

Thus, using (39) and (43), the SEP for $M$-PAM can be expressed as

$$
\mathcal{P}_{P A M}^{s}=\delta \sum_{k=0}^{\infty} c_{k} L\left(a, 2 \beta, \frac{n}{2}+k\right) .
$$

Additionally, from (29) and (43), after some manipulations, the truncation error bound of $\mathcal{P}_{P A M}^{S}$ can be expressed in closed-form as

$$
\mathcal{E}_{P A M}^{s}=\delta c_{0} \sum_{i=1}^{n / 2} \Delta_{i} \mathcal{L}\left(a, 2 \beta, \xi_{2 i-1}, N+\frac{n}{2}-1\right)
$$


where

$$
\mathcal{L}(a, b, \rho, m)=\frac{L(a, b /(1-\rho), 1)}{1-\rho}-\sum_{l=0}^{m} \rho^{l} L(a, b, l+1)
$$

which can be also very easily evaluated.

$M$-QAM: Employing a rectangular QAM scheme with $M_{I}$ amplitude levels for the in-phase channel and $M_{Q}$ levels for the quadrature channel, $P(\gamma)$ becomes

$$
\begin{aligned}
P(\gamma) & =\delta_{I} \mathcal{Q}\left(a_{I} \sqrt{\gamma}\right)+\delta_{Q} \mathcal{Q}\left(a_{Q} \sqrt{\gamma}\right) \\
& -\delta_{I} \delta_{Q} \mathcal{Q}\left(a_{I} \sqrt{\gamma}\right) \mathcal{Q}\left(a_{Q} \sqrt{\gamma}\right)
\end{aligned}
$$

where the coefficients $\delta_{I}, a_{I}$ and $\delta_{Q}, a_{Q}$ are calculated by setting $M=M_{I}$ and $M=M_{Q}$ in (42) respectively. Thus, from [18] the symbol error probability becomes

$$
\begin{aligned}
\mathcal{P}_{Q A M}^{s} & =\delta_{I} \sum_{k=0}^{\infty} c_{k} L\left(a_{I}, 2 \beta, \frac{n}{2}+k\right) \\
& +\delta_{Q} \sum_{k=0}^{\infty} c_{k} L\left(a_{Q}, 2 \beta, \frac{n}{2}+k\right) \\
& -\delta_{I} \delta_{Q} \sum_{k=0}^{\infty} c_{k} H\left(a_{I}, a_{Q}, 2 \beta, \frac{n}{2}+k\right)
\end{aligned}
$$

with

$$
H\left(a_{I}, a_{Q}, b, m\right)=\frac{1}{4}-\left[v\left(a_{I}, a_{Q}, b, m\right)+v\left(a_{Q}, a_{I}, b, m\right)\right]
$$

and

$$
\begin{aligned}
v\left(a_{1}, a_{2}, b, m\right) & =\frac{1}{\sqrt{\pi}} \sum_{n=0}^{m-1} \frac{2^{n-1} a_{1} \sqrt{b} \Gamma(n+3 / 2)}{\left(a_{1}^{2} b+2\right)^{n+1 / 2} n !(1+2 n)} \\
& -\frac{1}{4 \pi} \sum_{n=0}^{m-1} \frac{b a_{1} a_{2} 2^{n+1}}{\left(a_{1}^{2} b+2+a_{2}^{2} b\right)^{n+1}} \\
& \times{ }_{2} F_{1}\left(1,1+n ; \frac{3}{2} ; \frac{a_{2}^{2} b}{a_{1}^{2} b+2+a_{2}^{2} b}\right) .
\end{aligned}
$$

where ${ }_{2} F_{1}(\cdot, \cdot ; \cdot ; \cdot)$ is the Gauss Hypergeometric function [16, eq. (15.1.1)]. In the case of a square QAM, (50) can be simplified as [9, eq. (5.30)]. Working in a similar way and using (40) and (48) the following bound for the SEP series truncation error can be found:

$$
\begin{aligned}
\mathcal{E}_{Q A M}^{s} & =c_{0} \delta_{I} \sum_{i=1}^{n / 2} \Delta_{i} \mathcal{L}\left(a_{I}, 2 \beta, \xi_{2 i-1}, N+\frac{n}{2}-1\right) \\
& +c_{0} \delta_{Q} \sum_{i=1}^{n / 2} \Delta_{i} \mathcal{L}\left(a_{Q}, 2 \beta, \xi_{2 i-1}, N+\frac{n}{2}-1\right) \\
& -c_{0} \delta_{I} \delta_{Q} \sum_{i=1}^{n / 2} \Delta_{i} \mathcal{H}\left(a_{I}, a_{Q}, 2 \beta, \xi_{2 i-1}, N+\frac{n}{2}-1\right)
\end{aligned}
$$

where

$$
\begin{aligned}
\mathcal{H}\left(a_{I}, a_{Q}, b, \rho, m\right) & =\frac{H\left(a_{I}, a_{Q}, b /(1-\rho), 1\right)}{1-\rho} \\
& -\sum_{l=0}^{m} \rho^{l} H\left(a_{I}, a_{Q}, b, l+1\right) .
\end{aligned}
$$

$M$-PSK: For the case of $M$-PSK it is known that [9]

$$
P(\gamma)=\frac{1}{\pi} \int_{0}^{(M-1) \pi / M} \exp \left(-\gamma \log _{2} M \frac{g_{P S K}}{\sin ^{2} \theta}\right) d \theta
$$

with $g_{P S K}=\sin ^{2}(\pi / M)$. It can then be shown that the SEP is expressed as:

$$
\mathcal{P}_{P S K}^{s}=\sum_{k=0}^{\infty} c_{k} J(M, 2 \beta, n / 2+k)
$$

where $J(M, b, m)$ can be found in [9, eq. (8.115)]. Similarly, the truncation error bound on the SEP series is written as

$$
\begin{aligned}
\mathcal{E}_{P S K}^{S} & =\sum_{i=1}^{n / 2} c_{0} \Delta_{i} \frac{J\left(M, 2 \beta /\left(1-\xi_{2 i-1}\right), 1\right)}{1-\xi_{2 i-1}} \\
& -\sum_{i=1}^{n / 2} c_{0} \Delta_{i} \sum_{l=0}^{N+n / 2-1} \xi_{2 i-1}^{l} J(M, 2 \beta, l+1) .
\end{aligned}
$$

2) BEP Expressions: In the following, BEP expressions and the corresponding truncation error bounds are derived for various modulation schemes.

$M$-PAM BEP: For the calculation of BEP for an $M$-PAM system with Gray coding, $P(\gamma)$ is defined as [19]

$$
P(\gamma)=\frac{1}{\log _{2} M} \sum_{j=1}^{\log _{2} M} P_{b}(j, \gamma, M)
$$

where

$$
P_{b}(j, \gamma, M)=\frac{2}{M} \sum_{i=0}^{\left(1-2^{-j}\right) M-1} \delta_{M}(i, j) \mathcal{Q}\left(a_{i} \sqrt{\gamma}\right)
$$

with

$$
a_{i}=(2 i+1) \sqrt{\frac{6 \log _{2} M}{M^{2}-1}}
$$

and

$$
\delta_{M}(i, j)=(-1)^{\left\lfloor i 2^{j-1} / M\right\rfloor}\left(2^{j-1}-\left\lfloor\frac{i 2^{j-1}}{M}+\frac{1}{2}\right\rfloor\right)
$$

where $\lfloor\cdot\rfloor$ denotes the floor operator. Consequently, using (43) the BEP can be written as

$$
\mathcal{P}_{P A M}^{b}=\sum_{k=0}^{\infty} \frac{c_{k}}{\log _{2} M} \sum_{j=1}^{\log _{2} M} P_{b j}\left(2 \beta, \frac{n}{2}+k, M\right)
$$

with

$$
P_{b j}(2 \beta, m, M)=\frac{2}{M} \sum_{i=0}^{\left(1-2^{-j}\right) M-1} \delta_{M}(i, j) L\left(a_{i}, 2 \beta, m\right)
$$

and its truncation error bound becomes

$$
\begin{aligned}
\mathcal{E}_{P A M}^{b} & =\sum_{i=1}^{n / 2} \frac{c_{0} \Delta_{i}}{\log _{2} M} \sum_{j=1}^{\log _{2} M} \frac{P_{b j}\left(2 \beta /\left(1-\xi_{2 i-1}\right), 1\right)}{1-\xi_{2 i-1}} \\
& -\sum_{i=1}^{n / 2} \frac{c_{0} \Delta_{i}}{\log _{2} M} \sum_{l=0}^{N+n / 2-1} \xi_{2 i-1}^{l} \sum_{j=1}^{\log _{2} M} P_{b j}(2 \beta, l+1) .
\end{aligned}
$$


$M$-QAM BEP: Examining again the case of a rectangular QAM with $M_{I}$ amplitude levels for the $I$-channel and $M_{Q}$ levels for the $Q$-channel, $P(\gamma)$ can be expressed as [19]

$$
\begin{aligned}
P(\gamma)=\frac{1}{\log _{2}\left(M_{I} M_{Q}\right)} & {\left[\sum_{j=1}^{\log _{2} M_{I}} P_{M_{I} M_{Q}}\left(j, \gamma, M_{I}\right)\right.} \\
& \left.+\sum_{j=1}^{\log _{2} M_{Q}} P_{M_{I} M_{Q}}\left(j, \gamma, M_{Q}\right)\right]
\end{aligned}
$$

with

$$
P_{M_{I} M_{Q}}\left(j, \gamma, M_{0}\right)=\frac{2}{M_{0}} \sum_{i=0}^{\left(1-2^{-j}\right) M_{0}-1} \delta_{M_{0}}(i, j) \mathcal{Q}\left(a_{i} \sqrt{\gamma}\right) .
$$

In this case, the coefficients $a_{i}$ are computed using the expression

$$
a_{i}=(2 i+1) \sqrt{\frac{6 \log _{2}\left(M_{I} M_{Q}\right)}{M_{I}^{2}+M_{Q}^{2}-2}}
$$

while the coefficients $\delta_{M_{0}}$ are given by setting $M=M_{0}$ in (60) and the BEP becomes

$$
\begin{array}{r}
\mathcal{P}_{Q A M}^{b}=\sum_{k=0}^{\infty} \frac{c_{k}}{\log _{2}\left(M_{I} M_{Q}\right)}\left[\sum_{j=1}^{\log _{2} M_{I}} K_{j}\left(2 \beta, \frac{n}{2}+k, M_{I}\right)\right. \\
\left.+\sum_{j=1}^{\log _{2} M_{Q}} K_{j}\left(2 \beta, \frac{n}{2}+k, M_{Q}\right)\right]
\end{array}
$$

with

$$
K_{j}\left(2 \beta, m, M_{0}\right)=\frac{2}{M_{0}} \sum_{i=0}^{\left(1-2^{-j}\right) M_{0}-1} \delta_{M_{0}}(i, j) L\left(a_{i}, 2 \beta, m\right) .
$$

Again, the truncation error bound on the BEP series can be calculated using (40) as

$$
\begin{aligned}
\mathcal{E}_{Q A M}^{b} & =\frac{c_{0}}{\log _{2} M_{I} M_{Q}} \\
& \times \sum_{i=1}^{n / 2} \Delta_{i}\left[\sum_{j=1}^{\log _{2} M_{I}} \mathcal{K}_{j}\left(2 \beta, \xi_{2 i-1}, N+\frac{n}{2}-1, M_{I}\right)\right. \\
& \left.+\sum_{j=1}^{\log _{2} M_{I}} \mathcal{K}_{j}\left(2 \beta, \xi_{2 i-1}, N+\frac{n}{2}-1, M_{Q}\right)\right]
\end{aligned}
$$

where

$$
\begin{aligned}
\mathcal{K}_{j}\left(b, \rho, m, M_{0}\right) & =\frac{K_{j}\left(b /(1-\rho), l+1, M_{0}\right)}{1-\rho} \\
& -\sum_{l=0}^{m} \rho^{l} K_{j}\left(b, l+1, M_{0}\right) .
\end{aligned}
$$

$M$-PSK BEP: For $M$-PSK with Gray-coding, a close approximation for $P(\gamma)$ is [9]

$$
P(\gamma) \cong \frac{2}{\max \left(\log _{2} M, 2\right)} \sum_{i=1}^{\max (M / 4,1)} \mathcal{Q}\left(a_{i} \sqrt{\gamma}\right)
$$

with

$$
a_{i}=\sqrt{2 \log _{2} M} \sin \frac{(2 i-1) \pi}{M} .
$$

Hence, a useful approximation of the BEP for OSTBC over Hoyt fading channels is

$$
\mathcal{P}_{P S K}^{b} \cong \sum_{k=0}^{\infty} \frac{2}{\max \left(\log _{2} M, 2\right)} \sum_{i=1}^{\max (M / 4,1)} L\left(a_{i}, 2 \beta, m\right) .
$$

\section{B. Capacity Analysis}

The normalized capacity of an OSTBC MIMO system, expressed in bits/sec/Hz, is given by [4]

$$
C=R \log _{2}\left(1+\frac{\|\mathbf{H}\|_{F}^{2}}{R n_{t}} \gamma_{T}\right)
$$

where $\gamma_{T}$ is the SNR at the transmitter side. In the performance evaluation of wireless communication systems, the most commonly used capacity related criteria are information outage probability (IOP) and ergodic capacity.

1) Information Outage Probability: IOP is defined as the probability that a given transmission rate $C_{0}$ cannot be supported [20]. Thus, denoting IOP as $P_{\text {out }}\left(C_{0}\right)$, it holds that

$$
P_{\text {out }}\left(C_{0}\right)=\operatorname{Pr}\left(C<C_{0}\right)=\operatorname{Pr}\left(\|\mathbf{H}\|_{F}^{2}<H_{0}\right)
$$

where from (74)

$$
H_{0}=\frac{R n_{t}}{\gamma_{T}}\left(2^{C_{0} / R}-1\right) .
$$

Consequently, the IOP can be easily evaluated as

$$
P_{\text {out }}\left(C_{0}\right)=P_{Q}\left(H_{0}\right)
$$

where $P_{Q}(\cdot)$ is the CDF of the quadratic form $\|\mathbf{H}\|_{F}^{2}$ given by (8). Moreover, the truncation error bound for the IOP is computed using (31) as

$$
\varepsilon_{i o p}\left(C_{0}\right)=c_{0} \sum_{i=1}^{n / 2} \Delta_{i} G\left(H_{0}, \xi_{2 i-1}, \beta, N+\frac{n}{2}-1\right) .
$$

2) Ergodic capacity: Ergodic capacity $\langle C\rangle$ is defined as the average value of the capacity [4]. By denoting with $Q_{0}$ the quadratic form

$$
Q_{0}=\frac{\|\mathbf{H}\|_{F}^{2}}{R n_{t}} \gamma_{T}
$$

$\langle C\rangle$ is written as

$$
\langle C\rangle=R \int_{0}^{\infty} p_{Q_{0}}(x) \log _{2}(1+x) d x .
$$

The integral in (80) can be evaluated noting that [21]

$$
\begin{aligned}
\Psi(b, l) & =\frac{1}{(l-1) !} \int_{0}^{\infty} x^{l-1} \ln (1+x) \exp \left(-\frac{x}{b}\right) d x \\
& =\exp \left(\frac{1}{b}\right) \sum_{i=1}^{l} b^{i} \Gamma\left(-l+i, \frac{1}{b}\right)
\end{aligned}
$$


with $\Gamma(\cdot, \cdot)$ being the upper incomplete Gamma function [16, eq. (6.5.3)], thus yielding the following expression for the ergodic capacity

$$
\langle C\rangle=R \log _{2}(e) \sum_{k=0}^{\infty} c_{k} \frac{\Psi\left(2 \beta, \frac{n}{2}+k\right)}{(2 \beta)^{n / 2+k}}
$$

where $e$ is the Neper number. Using (81), the following bound on the truncation error for the ergodic capacity can be obtained

$$
\begin{aligned}
E_{\langle C\rangle} & =R \log _{2}(e) c_{0}\left[\sum_{i=1}^{n / 2} \Delta_{i} \frac{\Psi\left(2 \beta /\left(1-\xi_{2 i-1}\right), 1\right)}{2 \beta}\right. \\
& \left.-\sum_{i=1}^{n / 2} \Delta_{i} \sum_{k=0}^{N+n / 2-1} \xi_{2 i-1}^{k} \frac{\Psi(2 \beta, k+1)}{(2 \beta)^{k+1}}\right]
\end{aligned}
$$

which completes the analysis.

It is interesting to note that for high SNRs, simple approximate expressions for the various performance criteria can be obtained. As it will be shown in the next section, for high SNR the BEP, SEP and IOP truncation error bounds decrease very rapidly. Therefore, the number of series terms that would be sufficient for a prescribed accuracy is reduced. Based on this observation, for high SNR the series expressions for the BEP, SEP and IOP could be simplified and approximated by their first terms. For instance, from (49) the SEP of $M$-QAM would be expressed as follows:

$$
\begin{aligned}
\mathcal{P}_{Q A M}^{s} & \approx c_{0}\left[\delta_{I} L\left(a_{I}, 2 \beta, \frac{n}{2}\right)+\delta_{Q} L\left(a_{Q}, 2 \beta, \frac{n}{2}\right)\right. \\
& \left.-\delta_{I} \delta_{Q} H\left(a_{I}, a_{Q}, 2 \beta, \frac{n}{2}\right)\right] .
\end{aligned}
$$

\section{Performance Evaluation and Discussion}

In this section, performance evaluation results will be presented comparing the previously derived theoretical analysis with computer simulations. In these experiments, we have considered a $2 \times 2$ MIMO system with Alamouti space-time coding [4]. In Fig. 1, truncation error bounds of the chi-squared series PDF for two Hoyt fading scenarios are depicted. In the first scenario, the channel fading coefficients are quite similar, i.e., $\left(\Omega_{1,1}, q_{1,1}\right)=(0.25,0.9),\left(\Omega_{1,2}, q_{1,2}\right)=(0.25,0.8)$, $\left(\Omega_{2,1}, q_{2,1}\right)=(0.25,0.75),\left(\Omega_{2,2}, q_{2,2}\right)=(0.25,0.7)$, resulting in a small spread of the quadratic form parameters $\lambda_{i}$ in (5). In the second scenario, a large spread is obtained by selecting the Hoyt parameters as $\left(\Omega_{1,1}, q_{1,1}\right)=$ $(0.4,0.4),\left(\Omega_{1,2}, q_{1,2}\right)=(0.3,0.5),\left(\Omega_{2,1}, q_{2,1}\right)=(0.2,0.6)$, $\left(\Omega_{2,2}, q_{2,2}\right)=(0.1,0.8)$. In both cases, the values of the parameter $\beta$ have been chosen according to (10). Moreover, we have considered $N=15$ and $N=20$ for the first and second scenarios, respectively. It can be observed from Fig. 1 that in both fading scenarios the proposed bound is meaningful and much tighter than the bound derived in [3], with the latter appearing to be impractical for large spreads of the quadratic form parameters. In the remaining simulations presented below, a $2 \times 2$ Alamouti scheme following scenario 2 has been employed.

In Fig. 2, both theoretical and simulation curves of the SEP for various modulation schemes are depicted. The theoretical curves were obtained from (45), (49) and (55) for various

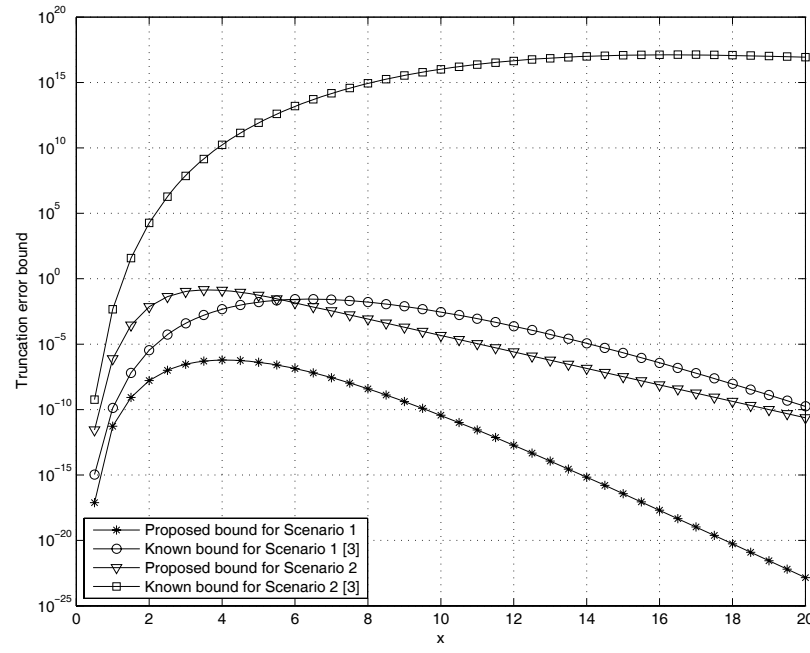

Fig. 1. Comparison of chi-squared series PDF truncation error bounds.

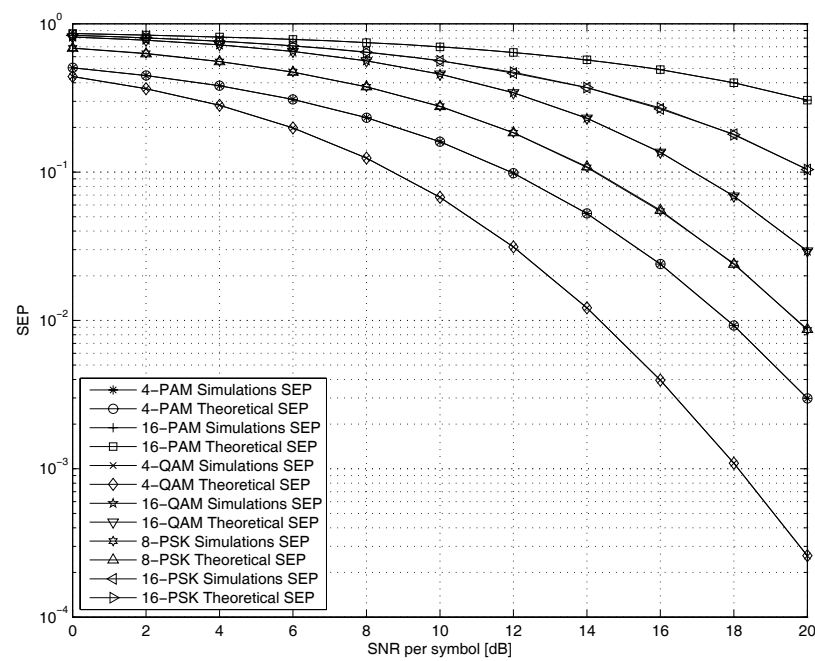

Fig. 2. SEP theoretical and simulations curves for several modulation schemes.

values of $M$. To obtain a truncation error bound that is at least one order of magnitude lower than the corresponding SEP value, a number of $N=25$ series terms was required. It has been observed through extensive computer simulations that one order of magnitude is a good compromise, which allows for sufficiently tight truncation error bounds and relatively small number of retained series terms. The almost perfect match of theoretical and simulation curves verifies the accuracy and fast convergence of the chi-squared series in representing the PDF.

The truncation error bound on the SEP series for 4-PAM and several values of $N$ given in (46) is shown in Fig. 3. It can be observed that a slight increase in the number of terms in the series leads to a significant improvement of the proposed bound. Moreover, it can be seen that for high SNR values the bound becomes negligibly small for all choices of $N$. This is also confirmed in Fig. 4 where the SEP series theoretical truncation error bound is plotted for several modulation schemes and $N=25$ according to (46), (52) and (56). It can be seen from the figure that as the SNR increases, the choice $N=25$ results in very small values 


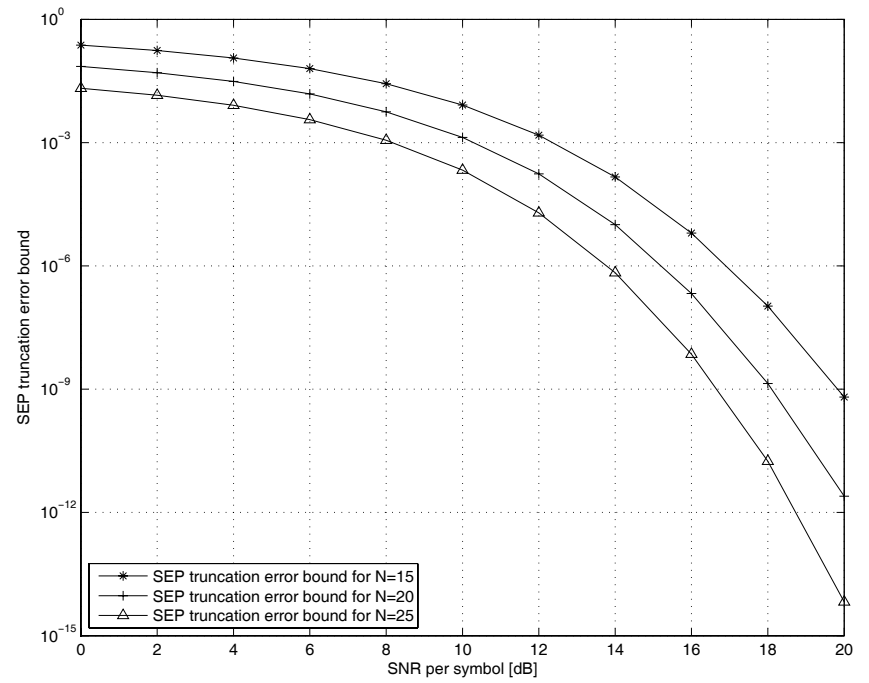

Fig. 3. Truncation error bound for the SEP series of 4-PAM for several values of $N$.

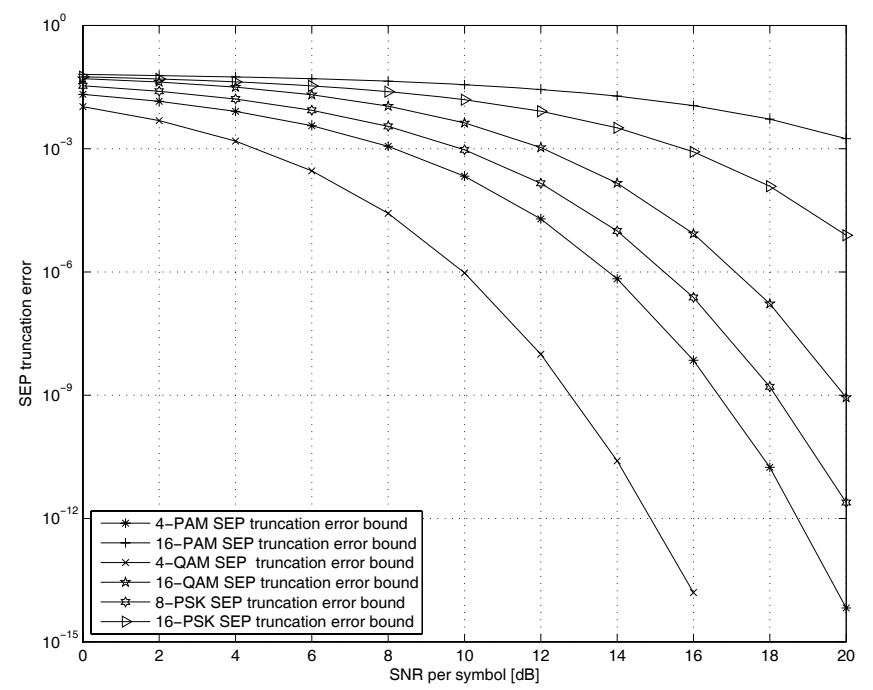

Fig. 4. SEP truncation error bound for several modulation schemes and $N=25$.

for the truncation error bound, that are multiple orders of magnitude lower than the calculated SEP. Thus, for high SNR values, a very small number of terms would be adequate for the accurate calculation of the SEP. Note that the same holds for all performance metrics considered in the sequel.

BEP theoretical and simulations results for various modulation schemes are illustrated in Fig. 5. Theoretical curves were obtained using (61), (67) and (73). The number of terms $N$ was taken equal to 20. Again, it can be observed that analytical and simulation curves almost coincide. In addition, the truncation error bounds on the BEP series for several PAM and QAM schemes as given by (63) and (69) are shown for $N=20$ in Fig. 6. Clearly the derived truncation error bounds make sense and thus can be considered as a fairly reliable criterion, when evaluating the accuracy of the chi-squared series expansion approach. It must be noticed that due to the different analytical expressions of various performance criteria and their corresponding truncation error bounds, a different number of series terms is required for each criterion in order

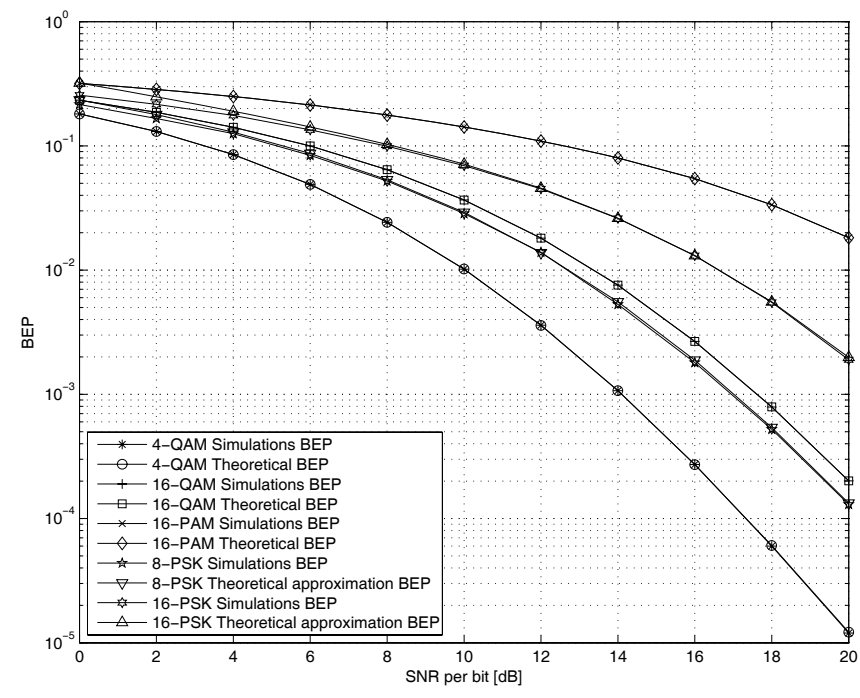

Fig. 5. BEP theoretical and simulation curves for PAM, QAM and PSK modulation schemes and $N=20$.

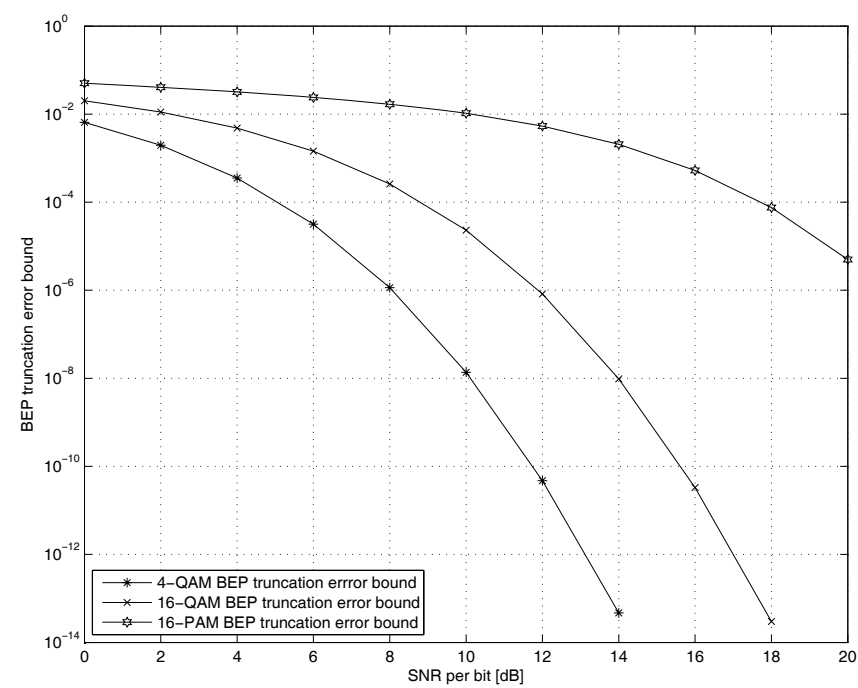

Fig. 6. BEP truncation error bounds for PAM and QAM and $N=20$.

to attain a predefined estimation accuracy.

In Fig. 7, the IOP is plotted as a function of the SNR for $C_{0}=3 \mathrm{bits} / \mathrm{sec} / \mathrm{Hz}$ and $N=25$. The theoretical curves for the IOP and the corresponding truncation error bound have been obtained form (77) and (78), respectively. We observe that there is a perfect match between theoretical and experimental results, while the bound is kept at least one order of magnitude lower than the IOP for all SNRs. Finally, theoretical and experimental ergodic capacity curves are presented in Fig. 8. In the same figure the theoretical truncation error bound of the ergodic capacity series is also plotted for $N=30$. The theoretical curves shown in the figure are obtained from (82) and (83) respectively. Again, simulations verify completely the presented analytical results.

\section{CONCLUSION}

This paper has presented a new perspective for the performance evaluation of OSTBC wireless communication systems. A basic property of OSTBC is that the SNR at the receiver 


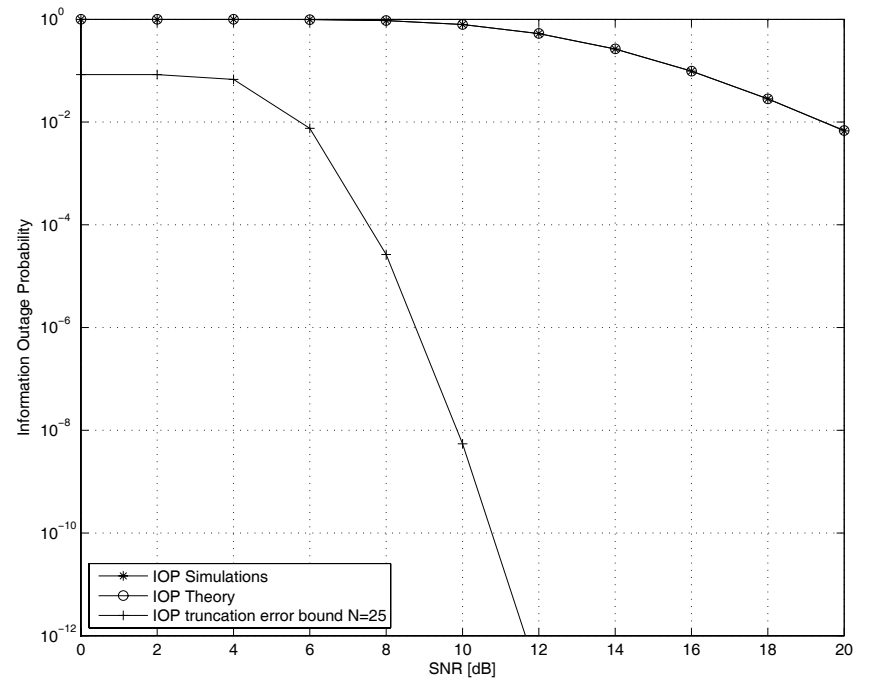

Fig. 7. Information outage probability versus SNR for $C_{0}=3 \mathrm{bits} / \mathrm{sec} / \mathrm{Hz}$ and $N=25$.

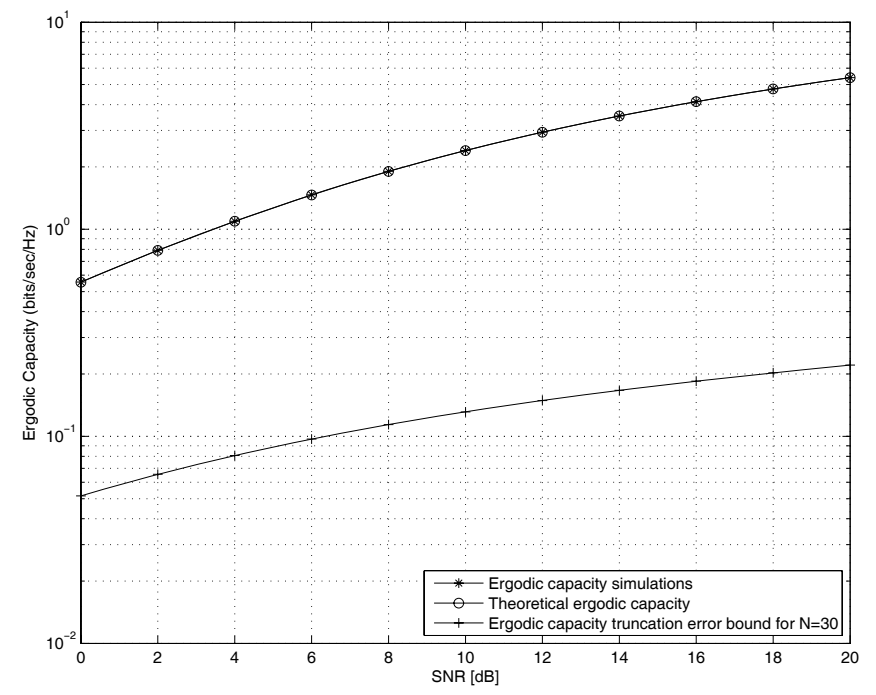

Fig. 8. Ergodic capacity for several values of the SNR and $N=30$.

for several fading models can be expressed as a quadratic form in normal RVs. Motivated by this, a statistical analysis of central, positive definite quadratic forms in normal RVs has been presented, stemming from the chi-squared series expansion approach. Novel, tight series truncation error bounds have been derived for both the PDF and CDF of central, positive definite quadratic forms in normal RVs. In an OSTBC wireless communication setup, such a form appears when the channel fading coefficients are Hoyt distributed. Based on this property, a detailed error probability and capacity analysis has been presented for OSTBC systems over Hoyt fading. Novel SEP, BEP, IOP and ergodic capacity infinite series expressions have been obtained, whose accuracy has been evaluated by newly derived truncation error bounds expressed in mathematically simple forms. Extensive computer simulated performance evaluations results have also been presented, which have completely verified the proposed analytical results.

\section{APPENDIX}

The purpose of this appendix is to derive an expression for the truncation error bound, when the parameters $\xi_{2 i-1}$ in (20) are non distinct. To this end, it is assumed that $S_{\zeta}=$ $\left\{\zeta_{1}, \zeta_{2}, \ldots, \zeta_{l}\right\}$ is the subset of distinct values of the set $S_{\xi}=$ $\left\{\xi_{1}, \xi_{3}, \ldots, \xi_{n-1}\right\}$ and $\left\{p_{1}, p_{2}, \ldots, p_{l}\right\}$ are the multiplicities of the elements of $S_{\zeta}$ with respect to $S_{\xi}$. Using $S_{\zeta}$, the MGF of $V$ is written as

$$
M_{V}(t)=\prod_{i=1}^{l} \frac{1}{\left(1-2 \zeta_{i} t\right)^{p_{i}}}=\sum_{i=1}^{l} \sum_{j=1}^{p_{i}} \frac{A_{i, j}}{\left(1-2 \zeta_{i} t\right)^{j}}
$$

where $A_{i, j}$ are easily computed [22]. The moments of $V$ will be given by

$$
E\left[V^{k}\right]=\sum_{i=1}^{l} \sum_{j=1}^{p_{i}} A_{i, j} \frac{\Gamma(j+k)}{\Gamma(j)}\left(2 \zeta_{i}\right)^{k} .
$$

It is thus easy to observe from (15) that in this case the bound on the truncation error is

$$
\begin{aligned}
e_{b}(x) & =\frac{c_{0} \exp \left(-\frac{x}{2 \beta}\right)}{2 \beta\left(\frac{n}{2}-1\right) !} \\
& \times \sum_{k=N+1}^{\infty} \sum_{i=1}^{l} \sum_{j=1}^{p_{i}} \frac{A_{i, j}(j)_{k} \zeta_{i}^{k}}{\left(\frac{n}{2}\right)_{k} k !}\left(\frac{x}{2 \beta}\right)^{n / 2+k-1}
\end{aligned}
$$

with $(\cdot)_{k}$ denoting the Pochhammer symbol. Thus, recalling the definition of the Hypergeometric function ${ }_{1} F_{1}(\cdot ; \cdot ; \cdot)$, [23, eq. (07.20.02.0001.01)],

$$
{ }_{1} F_{1}(a ; b ; z) \triangleq \sum_{k=0}^{\infty} \frac{(a)_{k} z^{k}}{(b)_{k} k !}
$$

$e_{b}(\cdot)$ becomes

$$
\begin{aligned}
e_{b}(x) & =\frac{c_{0}\left(\frac{x}{2 \beta}\right)^{n / 2-1} \exp \left(-\frac{x}{2 \beta}\right)}{2 \beta\left(\frac{n}{2}-1\right) !} \\
& \times\left[\sum_{i=1}^{l} \sum_{j=1}^{p_{i}} A_{i, j 1} F_{1}\left(j ; \frac{n}{2} ; \frac{\zeta_{i} x}{2 \beta}\right)\right. \\
& \left.-\sum_{i=1}^{l} \sum_{j=1}^{p_{i}} A_{i, j} \sum_{k=0}^{N} \frac{(j)_{k}\left(\frac{\zeta_{i} x}{2 \beta}\right)^{k}}{\left(\frac{n}{2}\right)_{k} k !}\right]
\end{aligned}
$$

Note that for integer $a, b$ and $a<b$, which holds in our case, the expression of the hypergeometric function ${ }_{1} F_{1}$ is simplified as in [23, eq. (07.20.03.0024.01)].

\section{ACKNOWLEDGMENT}

The authors would like to thank the anonymous reviewers and the editor for their valuable comments that have helped us improve the quality of the paper. 


\section{REFERENCES}

[1] A. M. Mathai and S. B. Provost, Quadratic Forms in Random Variables: Theory and Applications. New York: Marcel Dekker, 1992.

[2] H. Ruben, "Probability content of regions under spherical normal distributions, iv: the distribution of homogeneous and non-homogeneous quadratic functions of normal variables," The Annals of Mathematical Statistics, vol. 33, no. 2, pp. 542-570, June 1962.

[3] S. Kotz, N. L. Johnson, and D. W. Boyd, "Series representations of distributions of quadratic forms in normal variables: I. central case," The Annals of Mathematical Statistics, vol. 38, no. 3, pp. 823-837, June 1967.

[4] E. Larsson and P. Stoica, Space-Time Block Coding for Wireless Commun.. New York: CUP, 2003.

[5] I. Kim, "Exact BER analysis of OSTBCs in spatially correlated MIMO channels," IEEE Trans. Commun., vol. 54, no. 8, pp. 1365-1373, Aug. 2006.

[6] M. Dohler and H. Aghvami, "Information outage probability of distributed STBCs over Nakagami fading channels," IEEE Commun. Lett., vol. 8, no. 7, pp. 437-439, July 2004.

[7] G. Femenias, "BER performance of linear STBC from orthogonal designs over MIMO correlated Nakagami-m fading channels," IEEE Trans. Veh. Technol., vol. 53, no. 2, pp. 1074-1080, Mar. 2004.

[8] R. U. Nabar, H. Bolcskei, and A. Paulraj, "Diversity and outage performance in space-time block coded Ricean MIMO channels," IEEE Trans. Wireless Commun., vol. 4, no. 5, pp. 2519-2532, Sept. 2005.

[9] M. K. Simon and M.-S. Alouini, Digital Communication over Fading Channels, 2nd ed. New York: John Wiley and Sons, 2005.

[10] A. Anamalai, C. Tellambura, and V. K. Bhargava, "Simple and accurate methods for the outage analysis in cellular mobile radio systems-a unified approach," IEEE Trans. Commun., vol. 49, no. 2, pp. 303-316, Feb. 2001.

[11] J. Cheng and T. Berger, "Capacity of Nakagami- $q$ Hoyt fading channels with channel side information," in Proc. IEEE International Conf. Commun. Technol., vol. 55, Apr. 2003, pp. 1915-1918.

[12] R. M. Radaydeh, "Average error performance of $M$-ary modulation schemes in Nakagami- $q$ Hoyt fading channels," IEEE Commun. Lett., vol. 11, no. 3, pp. 255-257, Mar. 2007.

[13] B. Chytil, "The distribution of amplitude scintillation and the conversion of scintillation indices," J. Atmos. Terr. Phys., vol. 29, pp. 1175-1177, Sept. 1967

[14] S. Khatalin and J. P. Fonseka, "On the channel capacity in Rician and Hoyt fading environments with MRC diversity," IEEE Trans. Veh. Technol., vol. 55, no. 1, pp. 137-141, Jan. 2006.

[15] G. A. Ropokis, A. A. Rontogiannis, and P. T. Mathiopoulos, "Information outage probability of orthogonal space-time block codes over Hoyt fading channels," in Proc. IWSSC, vol. 2, Sept. 2006, pp. 135-139.

[16] M. Abramowitz and I. E. Stegun, Handbook of Mathematical Functions with Formulas, Graphs and Mathematical Tables. New York: Dover Publications, Inc., 1970.

[17] N. Sharma and E. Geraniotis, "Analyzing the performance of the spacetime block codes with partial channel state feedback," in Proc. IEEE Commun. Network. Conf., vol. 3, Sept. 2000, pp. 1362-1366.

[18] G. K. Karagiannidis, "On the symbol error probability of general order rectangular QAM in Nakagami-m fading," IEEE Commun. Lett., vol. 10, no. 11 , pp. 745-747, Nov. 2006.

[19] K. Cho and D. Yoon, "On the general BER expression of one- and twodimensional amplitude modulations," IEEE Trans. Commun., vol. 50, no. 7, pp. 1074-1080, July 2002.

[20] E. Telatar, "Capacity of multi-antenna gaussian channels," Eur. Trans. Telecom., vol. 10, no. 6, pp. 585-595, Dec. 1999.

[21] M. S. Alouini and A. J. Goldsmith, "Capacity of Rayleigh fading channels under different adaptive transmission and diversity combining techniques," IEEE Trans. Veh. Technol., vol. 48, no. 4, pp. 1165-1181, July 1999.
[22] G. K. Karagiannidis, N. C. Sagias, and T. A. Tsiftsis, "Closed-form statistics for the sum of squared Nakagami- $m$ variates and its applications," IEEE Trans. Commun., vol. 54, no. 8, pp. 1353-1359, Aug. 2006.

[23] Wolfram. (2008) The Wolfram functions site. [Online]. Available: http://functions.wolfram.com

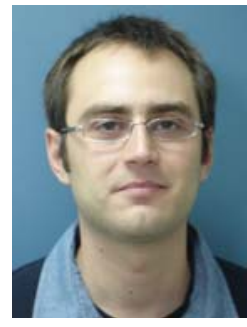

George A. Ropokis was born in Athens, Greece, on February 22, 1982. He received the diploma degree in computer engineering and informatics from the University of Patras, Greece in 2004, and the M.Sc. degree in mobile and satellite communications from the University of Surrey, UK. He is currently working towards a Ph.D. degree in wireless digital communication systems from the Computer Engineering and Informatics departemt, University of Patras. Since January 2006, he is also with the Institute for Space Applications and Remote Sensing, National Observatory of Athens, Greece. His research interests are focused on the performance analysis of wireless communications systems, MIMO and cooperative systems.

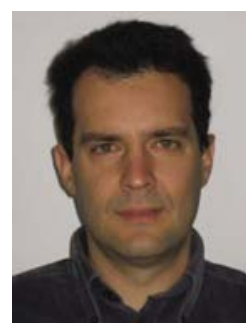

Athanasios A. Rontogiannis was born in Lefkada, Greece, in 1968. He received the Diploma degree in electrical engineering from the National Technical University of Athens, Greece, in 1991, the M.A.Sc. degree in electrical and computer engineering from the University of Victoria, Canada, in 1993, and the Ph.D. degree in communications and signal processing from the University of Athens, Greece, in 1997. From 1998 to 2003, he was with the University of Ioannina, where he was a lecturer in informatics since June 2000. In 2003 he joined the Institute for Space Applications and Remote Sensing, National Observatory of Athens, as a researcher on wireless communications. Dr. Rontogiannis has been a graduate and a post-graduate scholar of the Greek State Scholarship Foundation from 1994 to 1999. Currently, he serves as Associate Editor of the EURASIP Journal on ANDVANCES in Signal PROCESSING. His research interests are in the general areas of signal processing and wireless communications. $\mathrm{He}$ is a Member of the IEEE and the Technical Chamber of Greece.

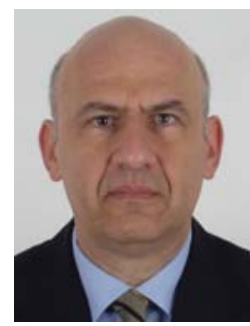

P. Takis Mathiopoulos (SM'04) received the $\mathrm{PhD}$ degree from the University of Ottawa. He is currently director of research at the Institute for Space Applications and Remote Sensing (ISARS) of the National Observatory of Athens (NOA), where he has established the Wireless Communications Research Group. Previously he was a Professor in the Department of Electrical and Computer Engineering at the University of British Columbia in Canada. His research interests are in the physical layer of terrestrial and satellite communication systems. Specific areas of research include transceiver structures, modulation and coding, MIMO, UWB, software radios, sensor networks, channel estimation and modelling, channel resource management techniques, and cross layer design. He has published more then 180 journal and conference papers in these areas. He has also delivered numerous invited presentations, including plenary lectures, and has taught many short courses all over the world. Dr. Mathiopoulos has served on the editorial board of many journals including the IEEE TRANSACTIONS ON COMMUNICATIONS. He has served as a TPC member for numerous IEEE Conferences and was a Vice Chair for the IEEE VTC2006-S and VTC2008-F. He has been an ASI Fellow, a Killam Research Fellow and a co-recipient of a best paper award (IEEE IWSSC 2008). 